\title{
Influence of Ultrasonication Parameters on Physical Characteristics of Olive Oil Model Emulsions Containing Xanthan
}

\author{
O. Kaltsa • I. Gatsi • S. Yanniotis • I. Mandala
}

Received: 4 June 2013 / Accepted: 13 January 2014 / Published online: 30 January 2014

(C) Springer Science+Business Media New York 2014

\begin{abstract}
Ultrasonic emulsification of $20-\mathrm{wt} . \%$ o/w emulsions ( $\mathrm{pH} 3.8$ ) containing a food-grade emulsifier (whey protein isolate, WPI, 2.7 wt.\%) and xanthan gum (XG, $0.25 \mathrm{wt} . \%)$ was performed. Time and amplitude of ultrasonic treatment changed in order to evaluate their influence on emulsion droplet size, viscosity, and stability (by multiple light scattering (MLS) profiles) during cold storage (10 days at $5{ }^{\circ} \mathrm{C}$ ). Ultrasonic treatment duration changed from 1 to 4 min at constant amplitude of $70 \%$. Considering the amplitude, intervals of $40,60,80$, and $100 \%$ were chosen, for a constant time of $1 \mathrm{~min}$. Similarly, time and amplitude conditions were used to treat solutions of XG of $1 \mathrm{wt} \%$ and evaluate their influence on viscosity and how that was related to the stability of the emulsion. Increase in sonication time from 1 to 4 min led to a significant oil droplet size decrease from 1.14 to $0.89 \mu \mathrm{m}$ (median droplet diameter). The viscosity of emulsions and XG solutions was highly influenced and considerably decreased with sonication time applied. At those conditions, an increase of backscattering was observed from 58.9 to $72.7 \%$ after 10 days of storage, meaning that more stable emulsions, thinner and of smaller oil droplet size were produced. A similar trend was observed when the amplitude was increased, but droplet size and creaming were always greater than those noticed by changing the sonication time. However, the rate of viscosity, droplet size, and stability change was greater by increasing the amplitude rather than by changing the sonication time.
\end{abstract}

Keywords o/w emulsion · Sonication parameters $\cdot$ Droplet size $\cdot$ Creaming $\cdot$ Viscosity

O. Kaltsa $\cdot$ I. Gatsi $\cdot$ S. Yanniotis $\cdot$ I. Mandala $(\bowtie)$

Department of Food Science and Technology, Agricultural

University of Athens, Athens, Greece

e-mail: imandala@aua.gr

\section{Introduction}

The use of ultrasonics in the food industry is gaining more interest. In-line implementation of ultrasonic processing is well established for the chemical and pharmaceutical industry and is being developed for food products such as fruit juices, mayonnaise, and tomato ketchup and dairy systems (Wu et al. 2000; Zisu et al. 2010).

Concerning emulsion technology, ultrasonic emulsification (USE) can offer several improvements compared with conventional high-shear methods or novel ones like microfluidization apart from power efficiency (Tang et al. 2013). This stands for production of oil droplets in the submicron/nanoscale (up to $100 \mathrm{~nm}$ ), improved stability over time, and need for small amounts of emulsifying agents (Chendke and Fogler 1975; Li and Chiang 2012). Although microfluidization has been found to be more efficient than ultrasound, it is considered less practicable with respect to production cost as well as equipment contamination (Karbstein and Schubert 1995; Freitas et al. 2006).

Despite the referred advantages, a number of restrictions considering USE have arisen. These involve demulsification (Canselier et al. 2002), deterioration in quality of edible oils and off-flavoring due to oxidation (Chemat et al. 2004; Gharibzahedi et al. 2012), and polymer degradation. Although there are many reports on ultrasonic degradation of polysaccharides (Lorimer et al. 1995; Tayal and Khan 2000; Wasikiewicz et al. 2005; Goodwin et al. 2011), these findings focus on rheological properties as affected by molecular weight reduction; thus, information are provided in terms of gum fractionation and viscosity tailoring. Therefore, there are only limited data to our knowledge relating the effect of gum viscosity decrease on emulsion stability (Vinod and Sashidhar 2009; Karaman et al. 2012; Ansari et al. 2012). On the other hand, sonication conditions' choice seems quite 
difficult, when food is treated and viscosity influences process and further product quality.

Optimization of sonication conditions deals with a careful determination and further choice of the variables influencing cavitation. These variables are related to the reaction medium (e.g., viscosity and vapor pressure), to the reaction conditions (e.g., frequency, power but temperature control as well), and to the type of the sonic system (e.g., frequency range) including the choice of the chemical reactor (e.g., horn shape and dimensions) (Santos et al. 2009). In the present research, some reaction conditions were changed (amplitude and sonication time), keeping sample formula constant and using the same sonic system. Amplitude choice in a sonication process is critical, because it is proportional to the intensity of sonication and its increase is related to an increase in the sonochemical effects. A minimum amplitude is required to achieve the cavitation threshold, whereas high amounts can lead to rapid deterioration of the ultrasonic transducer resulting in liquid agitation instead of cavitation and in poor transmission of the ultrasound through the liquid media. However, high-viscosity samples may require higher amplitude in order to obtain the necessary mechanical vibrations and to promote cavitation in the sample thereafter (Santos et al. 2009).

The type of cavitation is also related to the ultrasonic intensity and consequently to the amplitude applied. When intensity exceeds $10 \mathrm{~W} / \mathrm{cm}^{2}$, a transient cavitation occurs. In this range, bubbles expand through a few acoustic cycles to a radius of at least twice their initial size before collapsing violently on compression (Santos et al. 2009). On the other hand, cavitation bubbles' number and collapse are influenced by the temperature of the reaction medium. Temperature affects the vapor pressure, surface tension, and viscosity of the liquid medium (Muthukumaran et al. 2006). In a higher temperature, cavitation bubbles form more easily, because of medium viscosity decrease, but their collapse may be cushioned or dampened by the high vapor pressure (Muthukumaran et al. 2006; Zhou and Shi 2006). Thus, there is an optimum temperature at which viscosity is low enough to enhance cavitation bubble formation, yet the temperature is low enough to avoid the dampering effect (Moncada Reyes 2011).

In food systems, when ingredients degradation and structure change may occur by small changes in the ultrasonication process, a careful choice of process parameters helps to gain scientific and technological knowledge, and also to prevent undesirable quality effects. In this research, different sonication parameters (time and amplitude) were applied on a whey protein emulsion containing olive oil and xanthan gum produced in a batch-type process. Extra virgin olive oil was selected for nutritional reasons and health benefits related to its composition in combination with the fact that presents great stability under ultrasonication. No oxidation or hydrolysis effects were observed when ultrasounds $(20 \mathrm{kHz}, 120 \mathrm{~s})$ were applied during extraction of oil procedure from olive paste (Jiménez et al. 2007). Additionally, in comparison with other edible oils that are primarily used in salad dressings such as corn and sunflower oil, olive oil presented the highest absence of off-flavoring after sonication, which is related to the composition of the unsaturated fatty acids.

The formulation of these model o/w emulsions resembles that of a low fat and cholesterol-free mayonnaise or salad dressing. The main objective of this study was to establish an optimum of sonication conditions that lead to maximum stability emphasizing on gum viscosity characteristics and droplet size. For this reason, experimental findings relating gum viscosity, emulsion stability, and droplet size with ultrasonic parameters are discussed.

\section{Materials and Methods}

Whey protein isolate (WPI) Lacprodan DI-9224 was kindly provided by Arla (Arla Foods Ingredients, Amba-Denmark). The composition of the WPI was $92 \pm 2 \%$ protein, $0.2 \%$ maximum amounts of fat, $4.5 \%$ ash, and $0.2 \%$ lactose as stated by the manufacturer. Xanthan gum (XG) was obtained from Sigma (St. Louis, MO, USA). Virgin olive oil Altis (Elais Unilever, Greece) was purchased from a local store. Citric acid, phosphate, and sodium azide were purchased from Fluka (Fluka Chemie AG, Buchs, Switzerland).

\section{Emulsion Preparation}

WPI stock solution of $10 \mathrm{wt} \%$ in citric acid-phosphate buffer at $\mathrm{pH} 3.8$ was prepared by agitation with a magnetic stirrer for $90 \mathrm{~min}$. Sodium azide of $0.02 \mathrm{wt} . \%$ was added to the aqueous solutions as an antibacterial agent. XG solution of $1 \mathrm{wt} . \%$ was prepared by hot stirring in a water bath at $90{ }^{\circ} \mathrm{C}$ for $90 \mathrm{~min}$. Thereafter, solutions were kept overnight at $5{ }^{\circ} \mathrm{C}$ to ensure complete hydration. Coarse emulsions containing 2.7 wt.\% WPI, 20 wt. \% extra virgin olive oil, and 0.25 wt. $\%$ XG of a total of $50 \mathrm{~g}$ were prepared by mixing appropriate amounts of the aqueous stock solutions and oil with a high-shear device Ultraturrax T25 device (IKA Werke, Staufen, Germany) at $13.500 \mathrm{rpm}$ for $2 \mathrm{~min}$. Small adjustments on emulsion $\mathrm{pH}$ were made with a few drops of $\mathrm{HCl} 1 \mathrm{M}$. Forty milliliters of the coarse emulsion was placed in a glass beaker (38-mm internal diameter) covered by ice to prevent overheating $\left(<50{ }^{\circ} \mathrm{C}\right)$. Secondary emulsions were produced following a batch-type sonication approach. The ultrasonic tip of a 13-mm diameter cylindrical titanium probe (VS $70 \mathrm{~T}$ ) was immersed in the center of the glass beaker at a repeatable depth of $1 \mathrm{~cm}$, and different sonication treatments were applied generated from an ultrasonic device (Sonopuls 3200, Bandelin Gmbh \& Co, Berlin, Germany) operating at a constant frequency of $20 \mathrm{kHz}$. The sonication device operates by controlling either 
the percentage of amplitude (100\% amplitude corresponding to $170 \mu \mathrm{m}_{\mathrm{ss}}$ for the specific probe used) or power $(150 \mathrm{~W}$ nominal maximum power). It also has the ability to display or monitor the energy input $(\mathrm{kJ})$ in the sample during sonication. Sonication treatments were applied by controlling the percentage of amplitude $(40,60,80$, and $100 \%)$ for constant time (1 $\mathrm{min})$ and time (1, 2, 3, and $4 \mathrm{~min})$ at constant amplitude (70\%). The $70 \%$ amplitude was identified as the maximum applicable percentage that could be used for elongated emulsification (up to $4 \mathrm{~min}$ ) in order to avoid overheating and subsequent protein denaturation. The nominal total energy applied on the system was recorded from the device readings, and a digital thermometer was used to measure the temperature of the emulsion at the end of the sonication.

\section{Droplet Size Evaluation}

Optical microscopy is arguably the most precise among the existing general methods for drop-size determination, but it is time consuming, so it is used mainly in scientific studies in which very accurate results are needed (Denkova et al. 2004). Oil droplet size measurements were performed by means of optical microphotography and image analysis software processing (Krstonošić et al. 2009; Tonon et al. 2011; Zúñiga et al. 2013). The final emulsions were diluted with the same buffer solution ( $\mathrm{pH} 3.8$ ) at a 20:1 ratio, and several micrographs were recorded from an optical microscope (Kruss Optronik, Germany) with a $\times 40$ magnification connected with a camera (SONY, Topica TP-1002DS). The oil droplets' diameters were measured with an image analysis software (Image-Pro Plus 7.0, Media Cybernetics, Rockville USA), and conversion of micrographs' pixels to micrometer was made by using the micrometer ruler of the microscope. In total, a number of 1,000-1,200 droplets were measured, and results are demonstrated as the arithmetic median $\left(d_{50}\right)$, the volume-surface mean diameter or Sauter diameter $\left(d_{32}=\Sigma n_{i} d_{i}^{3} / \Sigma n_{i} d_{i}^{2}\right)$ that represents the average size based on the specific surface per unit volume and better characterizes small and spherical particles (Zúñiga et al. 2013), and the equivalent volume-mean diameter or de Brouckere diameter $\left(d_{43}=\Sigma n_{i} d_{i}^{4} / \Sigma n_{i} d_{i}^{3}\right)$, where $n_{i}$ is the number of droplets of diameter $d_{i}$ (Galazka et al. 1996; Huang et al. 2001; Leroux et al. 2003; Tonon et al. 2011). The $d_{43}$ is more sensitive to the presence of large particles in an emulsion (Walstra 2003), so it is more sensitive to fat droplet aggregation (Huck-Iriart et al. 2011; Relkin and Sourdet 2005). Therefore, $d_{43}$ could give a good indication about droplet recoalescence. An analytical mathematical explanation for the above attribute of the de Brouckere mean diameter could be found in Rawle (2002). The procedure was at least duplicated with differently prepared samples.
Cold Storage Stability

Emulsion stability was evaluated with a vertical scan analyzer Turbiscan MA 2000 (Formulaction, Toulouse, France) during 10 days of storage at $5{ }^{\circ} \mathrm{C}$. This multiple light scattering device allows the optical characterization of any type of dispersion by using a mobile reading head composed of a transmitting NIR diode $(\lambda=850 \mathrm{~nm})$ and two synchronous detectors analyzing the transmitted (T) and backscattered (BS) light with acquisitions of every $40 \mu \mathrm{m}$. Emulsion samples of about $6 \mathrm{ml}$ were put in a glass tube and scanned from bottom to $80-\mathrm{mm}$ tube height on a daily basis in order to obtain backscattering and transmission profiles that allow the calculation of instability due to creaming, sedimentation, coalescence, and other phenomena. An analytical description of the Turbiscan methodology could be found in Mengual et al. (1999).

To observe a creaming phenomenon, different approaches can be used: (a) the variation of backscattering at the top or the bottom, (b) the phase thickness (delta $\mathrm{H}$ ) of the cream layer or the clear phase, and (c) the migration velocity of the clarification front.

In the present work, the BS profiles were used to monitor the creaming kinetics (clarification) at the bottom of the tubes (6-20-mm zone) during cold storage. This zone was not affected by other destabilization phenomena. The obtained data were expressed as a percentage of the average backscattering intensity (\% BS) and plotted against storage time.

\section{Viscosity Measurements}

Viscosity flow curves of $1 \% \mathrm{XG}$ aqueous solutions were obtained using a controlled stress SR-5 rheometer (Universal Stress Rheometer/Rheometrics Scientific, Inc., NJ) with plateplate geometry and a $0.2-\mathrm{mm}$ gap. The diameter of the upper plate was $20 \mathrm{~mm}$. The temperature was maintained at $25 \pm$ $0.2{ }^{\circ} \mathrm{C}$ by circulating water from a constant temperature circulator. Flow curves (steady shear measurements) were obtained under controlled stress in the range of $0.5-50 \mathrm{~Pa}$. All measurements were performed in freshly prepared samples before serum phase separation was observed. The linear part of the viscosity $(\eta)$ curves was fitted in a power-law or Herschel-Buckley model according to the maximum $R^{2}$ values per sample achieved:

$\eta=k \gamma^{n-1}$

$\eta=\frac{\tau_{o}}{\gamma}+k^{\prime} \gamma^{n-1}$

where, $\gamma$ is the shear rate, $\tau_{o}$ is the yield stress, $n$ is the flow behavior index, and $k, k^{\prime}$ are the consistency indices to obtain rheological $\left(n\right.$ and $\left.k, k^{\prime}\right)$ and statistical $\left(R^{2}\right)$ parameters. The $k$, 
$k^{\prime}, \tau_{o}$, and $n$ values derived from Eqs. 1 and 2 are reported as the average of 2-3 separately prepared samples.

\section{Statistical Analysis}

Statistical analysis of the results was performed with Statgraphics Centurion XV (Statgraphics, Rockville, MD, USA), and the F-test was applied in order to compare the mean values of emulsions and XG solutions properties at $95 \%$ level of confidence.

\section{Results and Discussion}

Sono-emulsification Conditions

Energy input and final temperature of the samples derived from the different treatments are presented in Table 1. It should be noted that the initial temperature of the samples was $\sim 10^{\circ} \mathrm{C}$. It is shown that by varying either the amplitude or the duration of sonication treatment, the energy released in the samples follows a linear behavior $\left(R^{2}=0.997\right.$ and 0.999$)$, while the temperature of the samples at the end of the emulsification was exponentially influenced $\left(R^{2}=0.997\right.$ and 0.999$)$ according to amplitude and time change. From the slope of the energy curves, it can be seen that the energy input rate on the emulsions is much faster by increasing the amplitude but the sonication time is restricted. As a consequence, the emulsification is conducted in much higher temperatures leading to heating of the emulsions up to $\sim 44{ }^{\circ} \mathrm{C}$ after 4 min at $70 \%$ amplitude. On the contrary, in all cases of amplitude increments between 40 and $100 \%$ and constant time, the final temperature was kept below $\sim 32{ }^{\circ} \mathrm{C}$. The effect of energy input and emulsification temperature (even though it was not kept constant in this batch-type method) on emulsion stability attributes is discussed in the following paragraphs.

Influence of Sonication Treatment on Emulsions and XG Solution Viscosity

Ultrasonication and other high-shear treatments such as highpressure homogenization have been reported to influence the flow behavior of hydrocolloid dispersions due to reduction of their molecular weight (Price 1990; Marcotte et al. 2001; Syed Gulrez et al. 2012). The degradation of the macromolecules is considered of "mechanochemical" nature (Striegel 2003), since the depolymerization reactions that lead to chain cleavage can follow distinct mechanisms such as homolytic (formation of free macroradicals), heterolytic (oppositively charged macroions), or intramolecular degradation (stable macrofragments) (Stellbrink et al. 1998). Ultrasonic degradation of XG solutions leads to samples characterized by a low polydispersity and a narrow range of molecular weight that can be a representative for the given sample (Milas et al. 1986). Apparent viscosities of XG solutions and emulsions as affected by sonication treatments are demonstrated in Fig. 1. Consistency $\left(k, k^{\prime}\right)$ and flow behavior $(n)$ indices for all samples at each amplitude-time level are presented in Table 2. Flow curves and flow parameters indicate that all samples exhibit shear-thinning behavior at all ultrasonic amplitudes and duration levels. The experimental results show that the viscosity of polymer solutions and final emulsions decreased with an increase in the ultrasonication time and amplitude and approached a limiting value after $4 \mathrm{~min}$ of sonication (Fig. 1, Table 2). Solutions of untreated 1-wt.\% $\mathrm{XG}$ solutions are characterized by high consistency $\left(k^{\prime}=\right.$ $\left.24.0 \mathrm{~Pa} \mathrm{~s}^{\mathrm{n}}\right)$ and low-flow behavior index $(n=0.181)$, which dramatically changed even when the mildest ultrasonication

Table 1 Energy input applied and temperature rise during emulsification by various sonication treatments

\begin{tabular}{|c|c|c|c|c|c|c|c|}
\hline Sonication treatment & Energy (kJ) & Ultrasonic intensity $\left(\mathrm{W} / \mathrm{cm}^{2}\right)$ & Equation & $R^{2}$ & Temperature $\left({ }^{\circ} \mathrm{C}\right)$ & Equation & $R^{2}$ \\
\hline \multicolumn{8}{|c|}{ Amplitude percentage (\%) } \\
\hline \multicolumn{8}{|c|}{ (at constant time $=1 \mathrm{~min}$ ) } \\
\hline 40 & $3.48 \pm 0.08$ & 43.72 & & & $16.00 \pm 0.94$ & & \\
\hline 60 & $5.00 \pm 0.24$ & 62.81 & & & $21.91 \pm 1.87$ & & \\
\hline 80 & $6.92 \pm 0.11$ & 86.94 & $\mathrm{E}=0.0832 \times$ Amp. +0.129 & 0.997 & $26.02 \pm 0.80$ & $\mathrm{~T}=1.142 \times$ Amp $^{0.717}$ & 0.997 \\
\hline 100 & $8.39 \pm 0.24$ & 105.40 & & & $31.20 \pm 0.36$ & & \\
\hline \multicolumn{8}{|l|}{ Time (min) } \\
\hline \multicolumn{8}{|c|}{ (at constant amplitude percentage $=70 \%$ ) } \\
\hline 1 & $6.03 \pm 0.09$ & 75.75 & & & $25.10 \pm 1.85$ & & \\
\hline 2 & $11.86 \pm 0.26$ & 74.50 & & & $33.55 \pm 1.76$ & & \\
\hline 3 & $17.63 \pm 0.16$ & 73.83 & $\mathrm{E}=5.696 \times$ Time +0.4148 & 0.999 & $39.60 \pm 0.26$ & $\mathrm{~T}=25.182 \times \mathrm{Time}^{0.407}$ & 0.999 \\
\hline 4 & $23.09 \pm 0.22$ & 72.52 & & & $44.03 \pm 0.47$ & & \\
\hline
\end{tabular}

$E$ energy (kJ), Amp. amplitude percentage (\%) 
Fig. 1 Influence of various sonication treatments on the flow behavior of XG of $1 \mathrm{wt} . \%$ solutions (black square untreated sample, as affected by amplitude (black circle up to white circle), or time (black triangle up to white triangle))

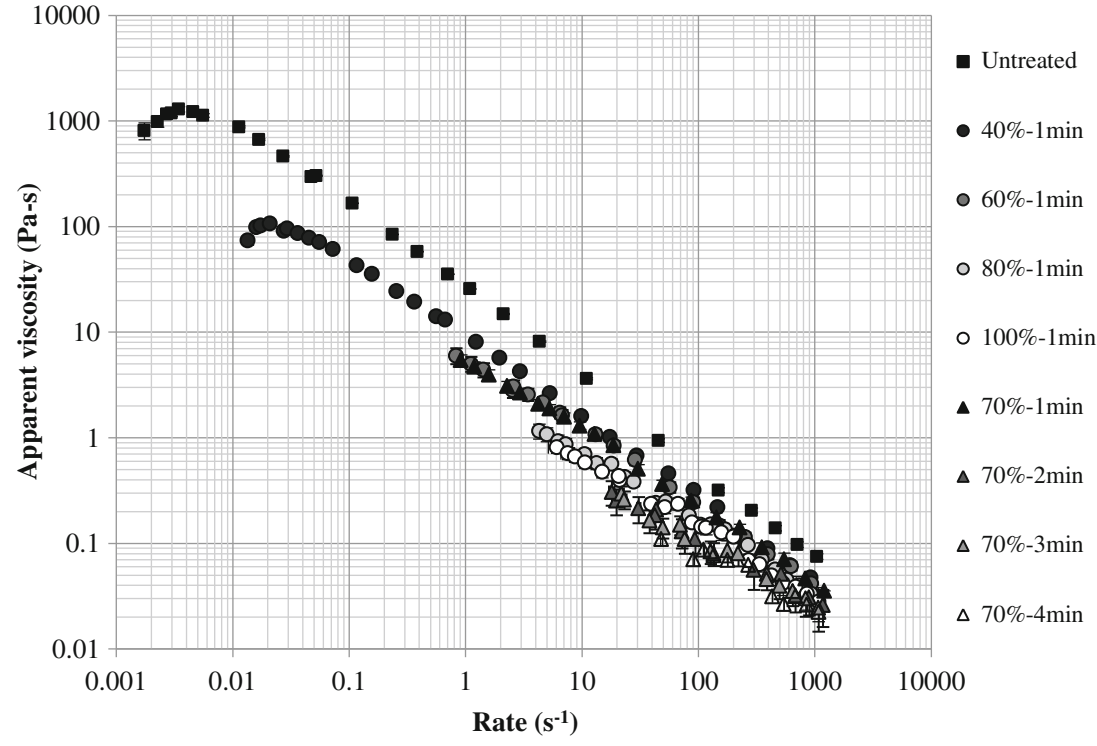

treatment (40\% amplitude, $1 \mathrm{~min})$ was applied $(p<0.05)$. Also, the yield stress, characteristic for xanthan solutions, disappears at amplitude values greater than $40 \%$ (Fig. 1). The sonication introduced a decreased shear-thinning behavior but still not Newtonian since maximum $n<1$ for both solutions and emulsions. In particular, emulsions presented greater $n$ values, indicative for a less shear-thinning behavior due to lower concentration of XG used in the formulation $(0.25 \%)$. This trend is in accordance with the findings of Tiwari et al. (2010), who studied the influence of highintensity ultrasound on the rheological characteristics of guar, xanthan, and pectin dispersions. They have concluded that the reduction of $k$ values in XG solutions depended strongly on intensity magnitude $\left(\mathrm{W} / \mathrm{cm}^{2}\right)$ and followed a linear decrease regression with increasing intensity level. Similar to our findings, as discussed below, the consistency index was reduced by $89.7 \%$ at the highest-intensity input $\left(10.1 \mathrm{~W} / \mathrm{cm}^{2}\right)$ compared with control samples.

In our experiments, it was found that ultrasonication time increase (at constant amplitude $70 \%$ ) had as a result a more pronounced effect on polymer viscosity compared with that obtained by amplitude increments (Table 2). Thus, in solutions, a consistency reduction of $77 \%$ was observed by amplitude increase from 40 to $100 \%$ and $81 \%$ by time increase from 1 to $4 \mathrm{~min}$, whereas in emulsion $k$, reduction was 45 and $52 \%$, respectively. Considering the flow index

Table 2 Flow behavior parameters of XG 1-wt.\% solutions and emulsions prepared with different sonication treatments

\begin{tabular}{|c|c|c|c|c|c|c|c|}
\hline & \multirow[b]{2}{*}{$\tau_{o}(\mathrm{~Pa})^{*}$} & \multicolumn{3}{|l|}{$1 \% \mathrm{XG}$ solution } & \multicolumn{2}{|l|}{ Emulsion } & \multirow[b]{2}{*}{$R^{2}$} \\
\hline & & $k, k\left(\mathrm{~Pa}-\mathrm{s}^{\mathrm{n}}\right)^{*}$ & $n(-)^{*}$ & $R^{2}$ & $k, k\left(\mathrm{~Pa}-\mathrm{s}^{\mathrm{n}}\right)^{*}$ & $n(-)^{*}$ & \\
\hline Untreated & $6.93 \pm 0.188^{b}$ & $24.00 \pm 0.247^{\mathrm{f}, * *}$ & $0.181 \pm 0.008^{\mathrm{a}, * *}$ & 0.996 & - & - & - \\
\hline \multicolumn{8}{|c|}{ Amplitude percentage (\%) } \\
\hline \multicolumn{8}{|c|}{$($ at constant time $=1 \mathrm{~min})$} \\
\hline 40 & $1.92 \pm 0.125^{\mathrm{a}}$ & $11.16 \pm 0.856^{\mathrm{e}, * *}$ & $0.196 \pm 0.012^{\mathrm{a}, * *}$ & 0.995 & $1.011 \pm 0.402^{\mathrm{c}}$ & $0.332 \pm 0.062^{\mathrm{a}}$ & 0.995 \\
\hline 60 & - & $4.37 \pm 0.949^{\mathrm{d}}$ & $0.309 \pm 0.014^{\mathrm{b}}$ & 0.988 & $0.903 \pm 0.181^{\mathrm{cd}}$ & $0.461 \pm 0.096^{\mathrm{bc}}$ & 0.976 \\
\hline 80 & - & $3.18 \pm 0.378^{\mathrm{cd}}$ & $0.331 \pm 0.024^{\mathrm{b}}$ & 0.975 & $0.730 \pm 0.067^{\mathrm{bcd}}$ & $0.477 \pm 0.015^{\mathrm{bc}}$ & 0.978 \\
\hline 100 & - & $2.58 \pm 0.269^{\mathrm{c}}$ & $0.354 \pm 0.011^{\mathrm{bc}}$ & 0.982 & $0.553 \pm 0.127^{\mathrm{ab}}$ & $0.490 \pm 0.028^{\mathrm{bc}}$ & 0.968 \\
\hline \multicolumn{8}{|l|}{ Time (min) } \\
\hline \multicolumn{8}{|c|}{ (at constant amplitude percentage $=70 \%$ ) } \\
\hline 1 & - & $4.12 \pm 0.580^{\mathrm{d}}$ & $0.308 \pm 0.022^{\mathrm{b}}$ & 0.996 & $0.855 \pm 0.148^{\mathrm{bcd}}$ & $0.413 \pm 0.052^{\mathrm{ab}}$ & 0.973 \\
\hline 2 & - & $2.38 \pm 0.408^{\mathrm{c}}$ & $0.359 \pm 0.016^{\mathrm{bc}}$ & 0.980 & $0.601 \pm 0.052^{\mathrm{abc}}$ & $0.514 \pm 0.018^{\mathrm{bc}}$ & 0.967 \\
\hline 3 & - & $1.49 \pm 0.216^{\mathrm{b}}$ & $0.420 \pm 0.020^{\mathrm{c}}$ & 0.966 & $0.524 \pm 0.107^{\mathrm{a}}$ & $0.521 \pm 0.024^{\mathrm{c}}$ & 0.945 \\
\hline 4 & - & $0.79 \pm 0.268^{\mathrm{a}}$ & $0.499 \pm 0.052^{\mathrm{d}}$ & 0.920 & $0.407 \pm 0.175^{\mathrm{a}}$ & $0.552 \pm 0.103^{\mathrm{c}}$ & 0.969 \\
\hline
\end{tabular}

*Values followed by the same letters at the same column are not significantly different $(p>0.05)$

**Values estimated by Herschel-Buckley model 
Fig. 2 Consistency index $(k)$ of XG of 1 wt.\% solutions (white triangle, white circle) and emulsions (black triangle, black circle) as a function of sonication energy applied. Circles represent amplitude (40-100 \%) and triangles time increments (14 min)

Fig. 3 a Surface $\left(d_{32}\right)$ and volume $\left(d_{43}\right)$ weighted diameters and arithmetic median diameter $\left(d_{50}\right)$ of freshly prepared emulsions as affected by various sonication treatments b Sauter mean $\left(d_{32}\right)$ diameters as a function of energy applied. Circles represent amplitude (40-100 \%) and triangles time increments (1$4 \mathrm{~min}$ ). Values followed by the same letters are not significantly different $(p>0.05)$
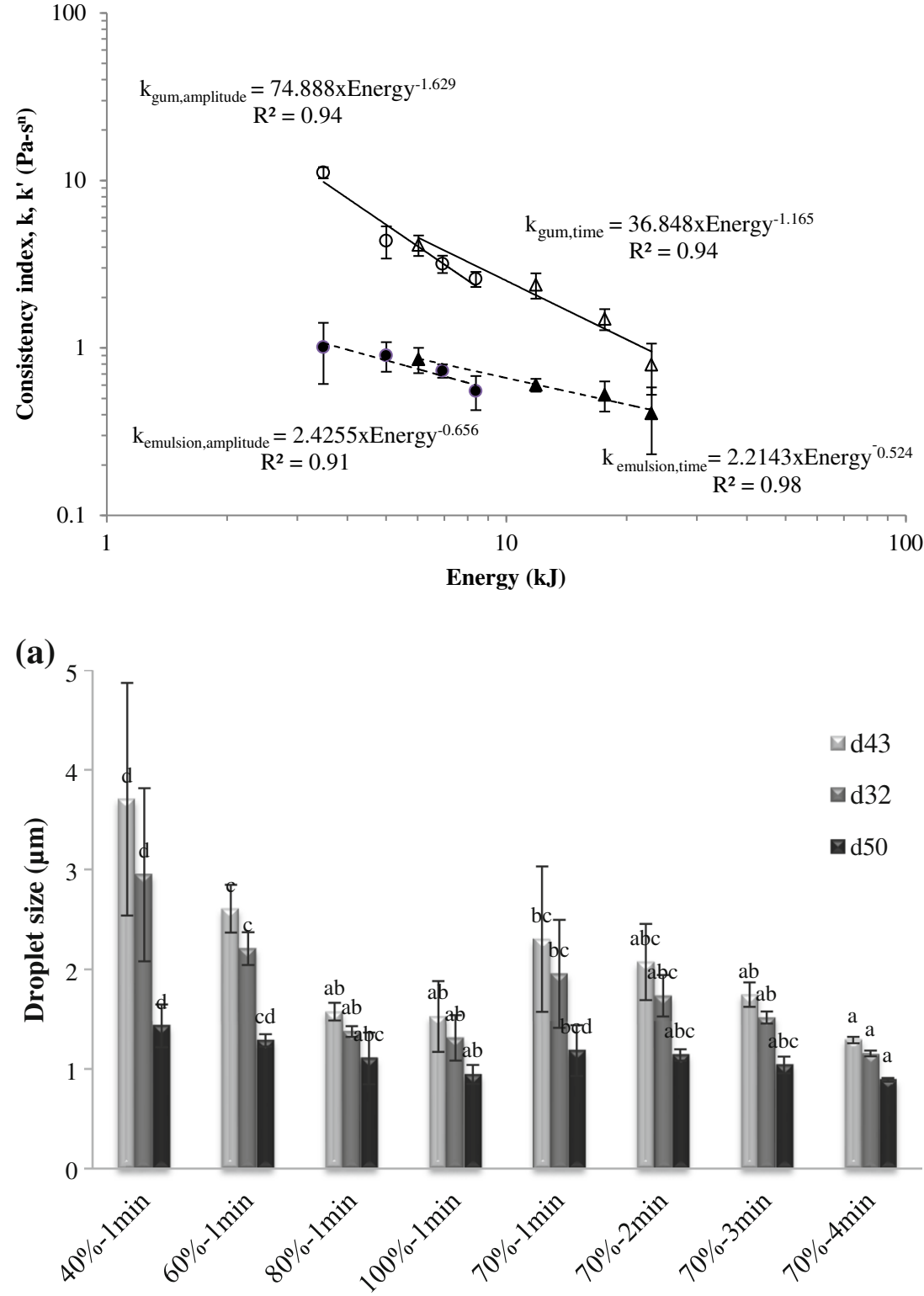

(b)

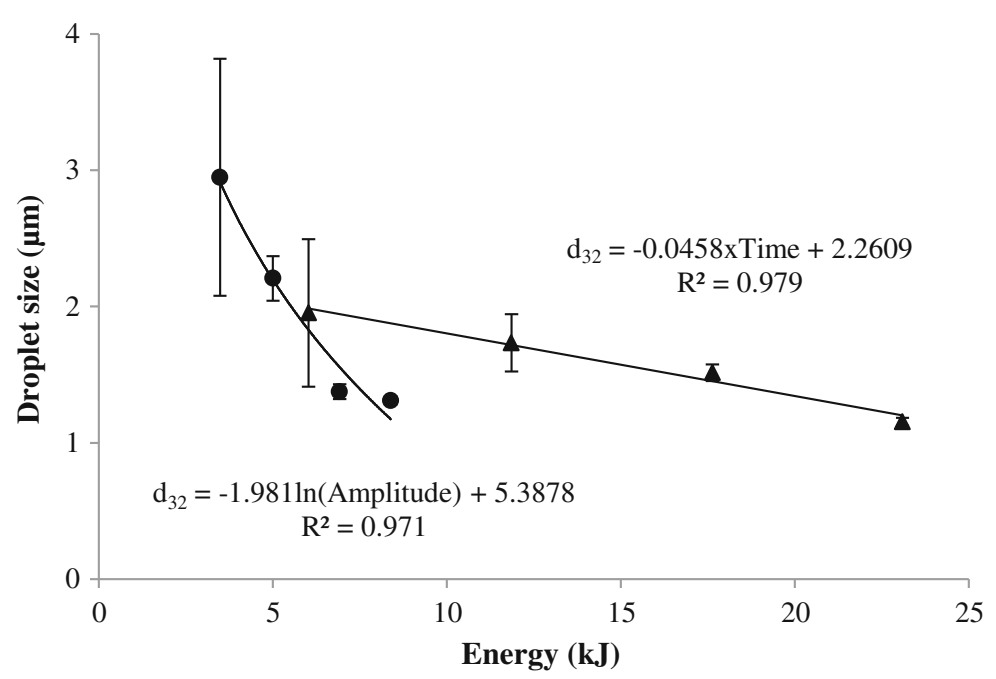


values, the greatest changes were observed for $\mathrm{XG}$ solutions sonicated for elongated time (4 min), for which $n=0.499$. Nevertheless, for emulsions treated with the maximum amplitude or time for which $n=0.490$ and 0.552 , respectively, no significant difference was found $(p>0.05)$.

In Fig. 2, data are presented as a function of energy applied on the system in order to better understand the influence of amplitude and sonication duration on sample consistency. Consistency values of gum solutions and emulsions followed a power-law decrease as a function of amplitude and time, as can be seen. It is also shown that the consistency change rate (slope) of xanthan solutions is much greater, almost double, by increasing the amplitude than by increasing the sonication time. The same trend is observed by comparing emulsions' consistency, although the slopes are similar. In the case of $n$ values, although a clear trend was identified as discussed above, the correlation of models was poor to demonstrate in most cases $\left(R^{2}<0.9\right)$.

Regarding the role of sonication temperature on viscosity properties over the range of $40-60{ }^{\circ} \mathrm{C}$, it is considered negligible (Karaman et al. 2012). However, in another research related to the effect of ultrasonics on chitosan fragmentation, it has been concluded that the chain scissions were strongly affected by sonication power as well as by the solution temperature (Kasaai et al. 2008). Nevertheless, the influence of temperature in the case of xanthan could be considered minor since it displays a structural stability over a wide range of temperature and shifts from an ordered to disordered structure at temperatures above $40{ }^{\circ} \mathrm{C}$ (Kang and Pettit 1993; Casas et al. 2000).

\section{Influence of Sonication Treatment on Droplet Size}

During the emulsification process, two different phenomena (droplet disruption and recoalescence) occur at the same time. The kinetics of each one individually affects the final droplet size of emulsions (Jafari et al. 2008). Thus, an optimum energy input should be established to avoid overprocessing regarding droplet size increase (Kentish et al. 2008). The influence of sonication treatment on droplet size according to amplitude (\%) and time increase is demonstrated in Figs. 3, 4, and 5.

As shown in Fig. 3, the particle diameters of emulsions were decreased upon increase of amplitude and sonication duration. These differences were more evident in the case of $d_{32}$ and $d_{43}$, whereas $d_{50}$ values did not decrease remarkably, and statistical differences were not noticed in most of the cases ( $p>0.05$ ). According to Behrend et al. (2000), a logarithmic reduction of $d_{32}$ occurs when increasing the energy density (energy per unit volume). In the present study, size reduction was also reduced in a logarithmic way when increasing the energy input by means of amplitude and a limiting value was reached after $\sim 7 \mathrm{~kJ}$ ( $80 \%$ amplitude; Fig. $3 \mathrm{~b}$ ). On the contrary, when longer sonication was applied at $70 \%$ amplitude, the diameter decrease followed a linear trend. This suggests that ultrasonication, at these experimental conditions, is more efficient in decreasing the polydispersity of the emulsions rather than remarkably minimizing the size of the smaller droplets in this system. Furthermore, samples prepared at $40 \%$ amplitude presented the highest values of $d_{43}$ compared with samples prepared with all other treatments applied $(p<0.05)$, meaning that overprocessing in terms of recoalescence was not observed even for samples treated with maximum amplitude or most elongated time. In another research, it has been suggested that a lower amplitude percentage would be more appropriate in order for the protein to better adsorb at the
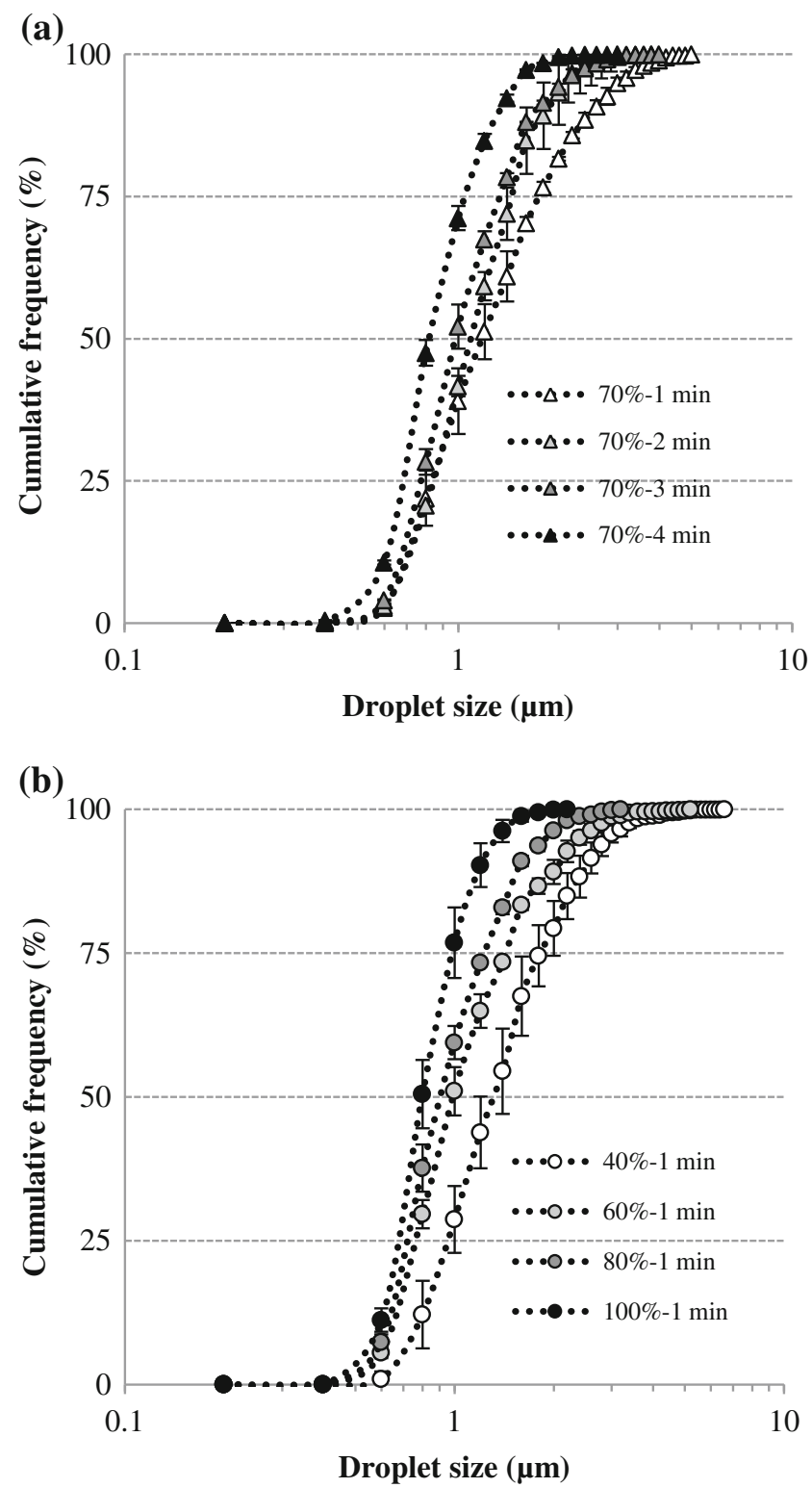

Fig. 4 Droplet size cumulative distributions of emulsions as affected by sonication a amplitude at constant time $1 \mathrm{~min}$ and $\mathbf{b}$ time at constant amplitude $70 \%$ 
interfacial area of droplet surface (Tang et al. 2012), thus decreasing more efficiently the final droplet size compared with higher amplitude. Apparently, this has not been our case, assuming that the emulsification time at $40 \%$ amplitude was restricted. An increase of amplitude from 40 to $100 \%$ was able to decrease the median diameter of oil droplets from 1.432 to $0.943 \mu \mathrm{m}$. At the same time, an increase in sonication time from 1 to $4 \mathrm{~min}$ led to a droplet size decrease from 1.141 to $0.891 \mu \mathrm{m}$, assuming that the increase in amplitude is more effective in reducing the average droplet size of the samples. This is in agreement with previous findings about consistency and flow index change by changing the amplitude. An explanation for the significant role of the amplitude change in droplet size is that as ultrasonication power is a measure of the pressure amplitude, an increase in the applied pressure amplitude leads to more intensive cavitation. Then, an increased number of bubbles are generated as a result of a more frequent liquid thread breaking and collapse. Additionally, the energy input in the system increases the temperature which facilitates the dispersion of both liquid phases into another by means of interfacial tension, viscosity, and Laplace pressure decrease, thereby smaller droplets are produced, whereas at lower amplitude, a wider droplet distribution is observed (Gaikwad and Pandit 2008).
It can be noted that by increasing the amplitude or sonication time, differences between the $d_{50}, d_{32}$, and $d_{43}$ values decrease; thus, similar values at 80 and $100 \%$ amplitude and after $4 \mathrm{~min}$ of sonication are presented $(p>0.05)$. Thus, upon increase of sonication duration, the standard deviations of diameter values were noticeably decreased. This phenomenon is in accordance with the findings of other research (Tang et al. 2013; Cucheval and Chow 2008; Li and Fogler 1978) and as mentioned, indicative of a polydispersity reduction. On the other hand, this trend was not clearly identified when amplitude was increased; therefore, the repeatability of experiments is lower when emulsification duration is restricted for $1 \mathrm{~min}$. It is obvious that increase in time and amplitude resulted in narrower droplet size distributions (Figs. 4 and 5). For presentation reasons only, data for 40 and $100 \%$ amplitude and 1 and 4-min treatments by means of frequency histograms are demonstrated (Fig. 5). Prolonged sonication induces sample mixing due to extended turbulent flow, and consequently, an effective disruption of most droplets is achieved. Therefore, the application of 4-min sonication resulted in finer emulsions with the minimum $d_{43}$ value, although the decrease rate of droplet size was much greater by amplitude change rather than by sonication time increase. Coupled with the temperature rise that occurs at these
Fig. 5 Distribution histograms of oil droplets of freshly prepared emulsions as affected by various sonication treatments. At the background: microphotographs processed by Image-Pro Plus 7.0. Scale bar at lower right represents $20 \mu \mathrm{m}$

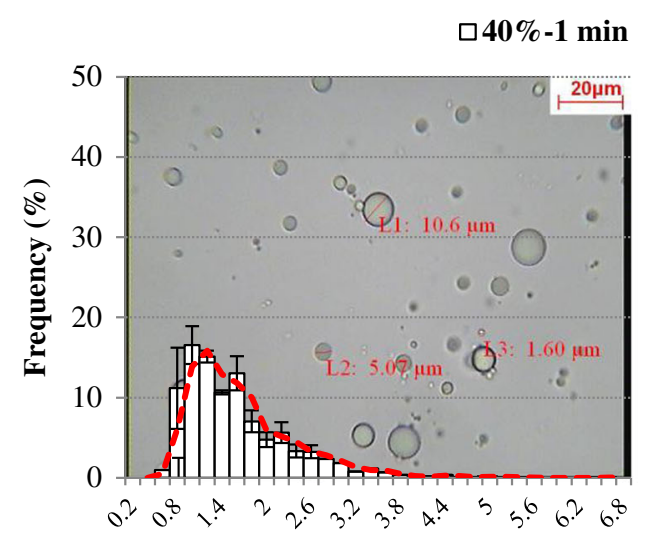

Droplet size $(\mu \mathrm{m})$

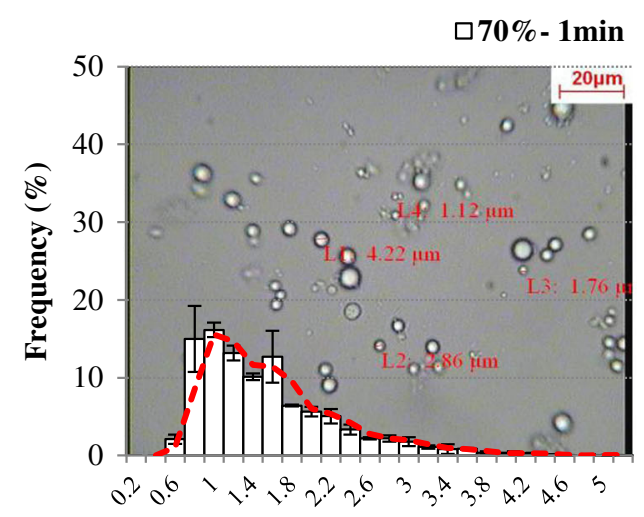

Droplet size $(\mu \mathrm{m})$
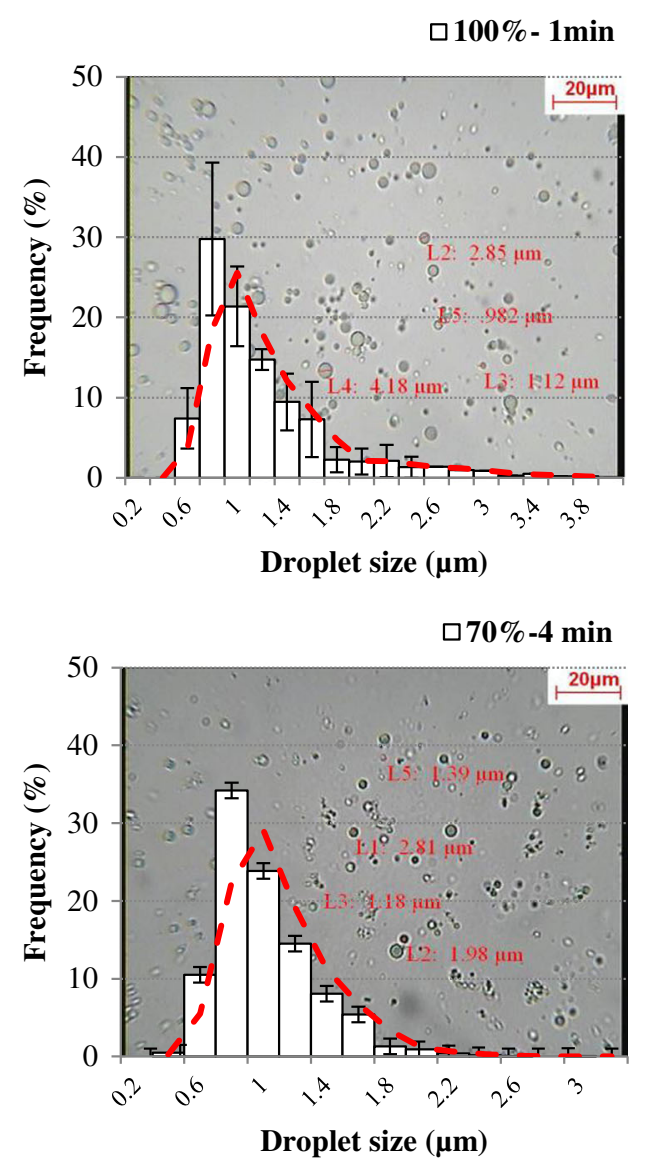
conditions, which reduces the minimum thermodynamic energy necessary for emulsification, the production of smaller droplets is facilitated (Canselier et al. 2002; McClements 2005).

Influence of Sonication Treatment on Emulsion Stability

Turbiscan measurements gave the time evolution over a 10day period of the backscattering intensity of emulsions along the tube height. The analysis of BS profiles as a function of time offers the advantage to allow a continued evaluation of the emulsion destabilization without previous dilution. The initial BS intensity $(t=0)$ is a value related to the size and density of particles in emulsions; BS enhances as the increase of the number of particles and decreases as a function of the size of individual droplets and/or presence of flocs (Palazolo et al. 2004, 2005). Hence, creaming kinetics can be easily monitored by the decrease of BS intensity at the bottom of the tube due to decrease of the concentration of the particles in this part (clarification) (Fameau 2012). It is also to be noted though that according to Mie's law, the BS intensity decreases when the droplet size increases only for particles with diameter larger than $0.5 \mu \mathrm{m}$ (Mengual 1999; Buron et al. 2004; Pizzino 2009).

The creaming of emulsions was strongly affected by sonication duration and amplitude, as seen in Fig. 6. In the case of amplitude (Fig. 6a), there was an improvement of emulsion stability expressed by the increased BS at the bottom zone, from 49.4 (40\% amplitude) to $64.2 \%$ (100\% amplitude) after 10 days of storage. Emulsions prepared by varying sonication time (at constant $70 \%$ amplitude) presented an even more pronounced stability (Fig. 6b). Hence, an increase in duration from 1 to $3 \mathrm{~min}$ led to an increase of the BS from 58.9 to $72.1 \%$ accordingly. Extended sonication time (4 min, $70 \%$ ) seems to have a negligible influence over stability, and this suggests a limiting boundary since the BS was only slightly increased to $72.7 \%(p>0.05)$. The marginal increase occurring in BS could be due to the decrease in viscosity of the continuous phase (Fig. 1, Table 2), since the oil droplet size was further decreased, and recoalescence (Jafari et al. 2006;
Fig. 6 Creaming kinetics (clarification) of emulsions during cold storage $\left(5^{\circ} \mathrm{C}\right)$ as affected by sonication a amplitude at constant time of 1 min and $\mathbf{b}$ time at constant amplitude $70 \%$. Values followed by the same letters are not significantly different $(p>0.05)$ (a)
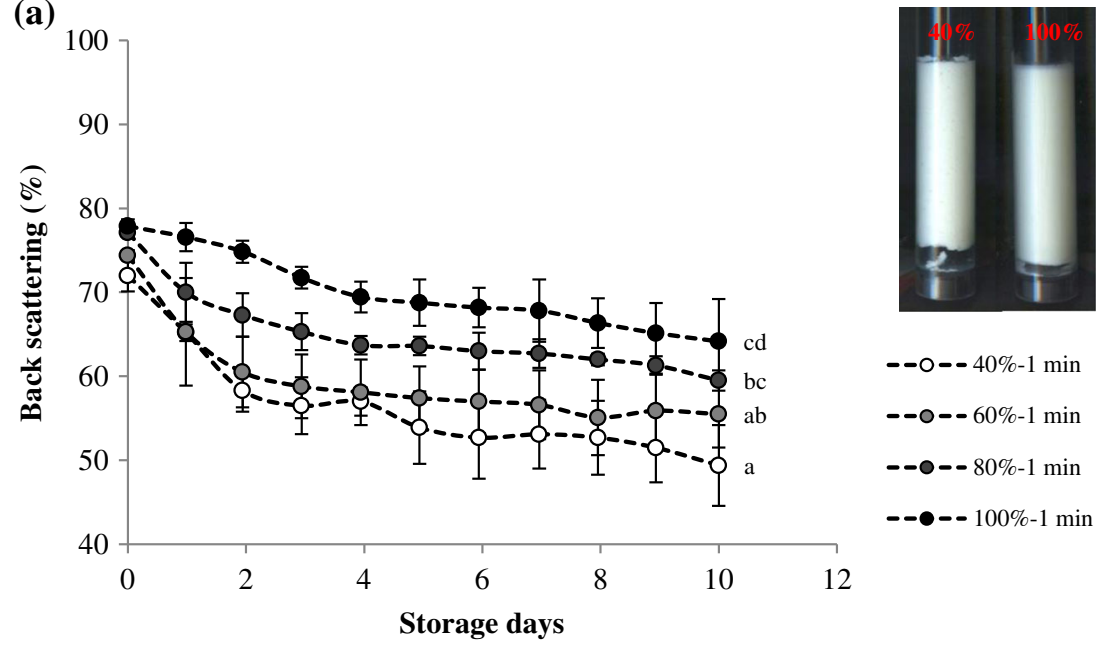

--0-- $40 \%-1 \mathrm{~min}$

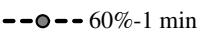

--0-- $80 \%-1 \mathrm{~min}$

--๑-- 100\%-1 min

12

(b)

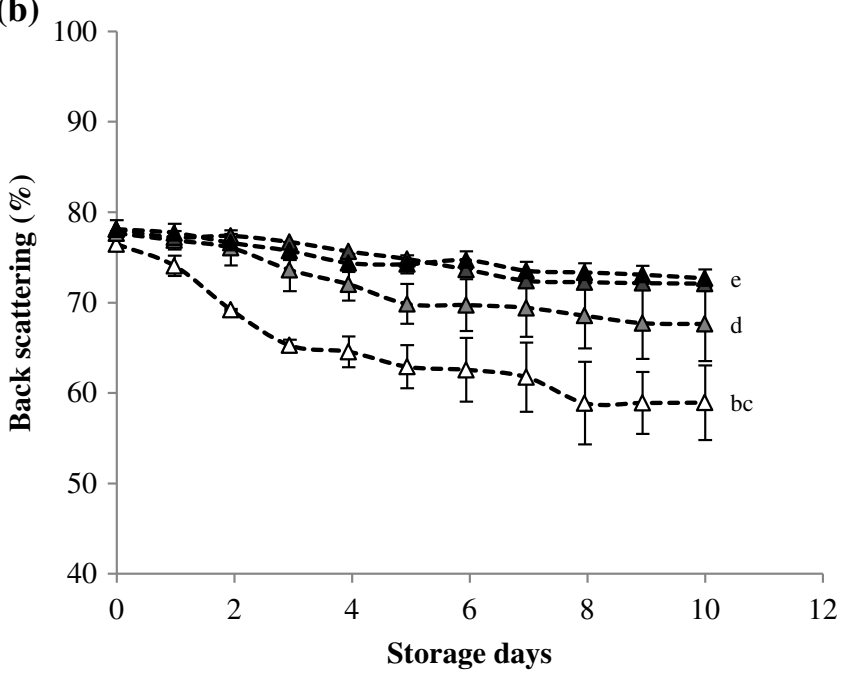

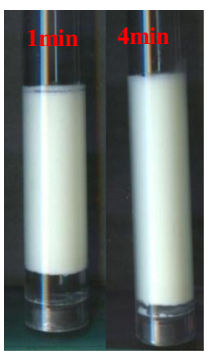

$--\Delta--70 \%-1 \min$

$--\Delta--70 \%-2 \min$

--^- $-70 \%-4 \min$
$--\Delta--70 \%-3 \mathrm{~min}$

Storage days 
Olson et al. 2004) was not observed. Stokes' law, $v_{s}=D^{2} g\left(\rho_{p}\right.$ $\left.-\rho_{f}\right) / 18 \mu$, where $v_{s}$ is the rising velocity of the droplet $(\mathrm{m} / \mathrm{s}), g$ is the gravitational constant $\left(9.81 \mathrm{~m} / \mathrm{s}^{-2}\right), D$ is the particle diameter $(\mathrm{m}), \rho_{p}$ is the density of settling particle and $\rho_{f}$ is the density of the continuous phase $\left(\mathrm{kg} / \mathrm{m}^{3}\right)$, and $\mu$ is the dynamic viscosity (Pa-s), provides a qualitative indication of the physical factors that influence the stability of an emulsion. The critical importance of particle size, occurring as a squared term, can be derived, as well as why emulsions are more stable when density differences are small, and when the viscosity of the continuous phase is high. However, in our case, even lowviscosity values resulted in improved stability, but there is a threshold value to assume that. This is because the relative flow of the particles under gravitational forces may break the emulsion, so stability is enhanced by small settling velocities. It should be noted that a small delay in creaming was observed when 3 and 4 min of sonication was applied; thus, emulsions started to destabilize after 3 days of storage. Creaming delay was observed in emulsions, where different amplitude increments were applied, only at $100 \%$ amplitude restricted at the first day of storage. In all the other cases of amplitude treatments, a sharp diminution of BS was observed even after $24 \mathrm{~h}$ of storage, corresponding to phase separation. Apart from decreasing the average droplet size, it has been shown that sonication treatment (40\% amplitude, $30 \mathrm{~s}, 20 \mathrm{kHz}$ ) was used to improve the stability of emulsions containing casein against creaming by disrupting the flocs formed upon acidification of emulsions from $\mathrm{pH} 7$ to $\mathrm{pH}$ 3, which enhanced electrostatic repulsion (Surh et al. 2006). Finally, among different treatments studied, the $100 \%$ for 1 -min sonication presented similar effects as the $70 \%$ for 2 min in terms of emulsion stability, XG solution and emulsion viscosity, and droplet size, owing to similar values of energy input.

\section{Conclusions}

An ultrasonication process application, in which the pressure amplitude (40-100\% for $1 \mathrm{~min}$ ) and the sonication time (70\% amplitude, 1-4 min) varied, resulted in emulsions treated in different ways. The increase in either of amplitude or time improved sample stability and led to a significant reduction of droplet size and viscosity. However, the impact of the varied parameters on emulsion quality became less pronounced as variable values approached maximum limits and differences among samples produced at higher amplitudes applied (80 and $100 \%$ ) or prolonged times ( 3 or $4 \mathrm{~min}$ ) were not great $(p<0.05)$ in most of the quality parameters measured. Optimum emulsification conditions were neither at highest amplitude values applied nor at the greatest sonication time used.

Amplitude increase at maximum did not reduce remarkably the droplet polydispersity, for which increase in time applied was required. Temperature increase was also noticed. Thus, a sonication time for $3 \mathrm{~min}$ is more appropriate at amplitude values of $70 \%$. Viscosity measurements showed that stability did not reduce at low-viscosity values resulted after longer treatments. However, a very low viscosity is responsible for recoalescence (70 \%, $4 \mathrm{~min}$ ) during storage. In terms of energy saving, the $100 \%$ for 1 -min treatment would be more preferable compared with $70 \%$ for $2 \mathrm{~min}$, since the time of sonication would be decreased by $50 \%$ and energy required by $29 \%$. These findings could be used for the successful production, using an ultrasonication process, of low-fat dressings based on olive oil and stored in a refrigerator.

Acknowledgments This research has been cofinanced by the European Union (European Social Fund-ESF) and Greek national funds through the Operational Program "Education and Lifelong Learning" of the National Strategic Reference Framework (NSRF)-Research Funding Program: Heracleitus II. Investing in knowledge society through the European Social Fund.

The authors would like to thank Arla Foods Hellas and Mr Andreas Andreou for kindly donating whey protein isolates.

\section{References}

Ansari, S. A., Matricardi, P., Di Meo, C., Alhaique, F., \& Coviello, T. (2012). Evaluation of rheological properties and swelling behaviour of sonicated scleroglucan samples. Molecules, 17, 2283-2297.

Behrend, O., Ax, K., \& Schubert, H. (2000). Influence of continuous phase viscosity on emulsification by ultrasound. Ultrasonics Sonochemistry, 7, 77-85.

Buron, H., Mengual, O., Meunier, G., Cayre, I., \& Snabre, P. (2004). Optical characterization of concentrated dispersions: applications to laboratory analyses and on-line process monitoring and control. Polymer International, 53, 1205-1209.

Canselier, J. R., Delmas, H., Wilhelm, A. M., \& Abismail, B. (2002). Ultrasound emulsification - an overview. Journal of Dispersion Science and Technology, 23, 333-349.

Casas, J. A., Mohedano, A. F., \& Garcia-Ochoa, F. (2000). Viscosity of guar gum and xanthan/guar gum mixture solutions. Journal of the Science of Food and Agriculture, 80, 1722-1727.

Chemat, F., Grondin, I., Costes, P., Moutoussamy, L., Shum Cheong Sing, A., \& Smadja, J. (2004). High power ultrasound effects on lipid oxidation of refined sunflower oil. Ultrasonics Sonochemistry, 11(5), 281-285.

Chendke, P. K., \& Fogler, H. S. (1975). Macrosonics in industry: 4 Ultrasonic innovations in the food industry: From the laboratory to commercial production. Chemical processing. Ultrasonics, 13, 3137.

Cucheval, A., \& Chow, R. C. Y. (2008). A study on the emulsification of oil by power ultrasound. Ultrasonics Sonochemistry, 15, 916-920.

Denkova, P. S., Tcholakova, S., Denkov, N. D., Danov, K. D., Campbell, B., Shawl, C., \& Kim, D. (2004). Evaluation of the precision of drop-size determination in oil/water emulsions by low-resolution NMR Spectroscopy. Langmuir, 20, 11402-11413.

Fameau, A. L., Ventureira, J., Novales, B., \& Douliez, J. P. (2012). Foaming and emulsifying properties of fatty acids neutralized by tetrabutylammonium hydroxide. Colloids and Surfaces A: Physicochemical and Engineering Aspects, 403(5), 87-95. 
Freitas, S., Hielscher, G., Merkle, H. P., \& Gander, B. (2006). Continuous contact- and contamination-free ultrasonic emulsification. Ultrasonics Sonochemistry, 13(1), 76-85.

Gaikwad, S. G., \& Pandit, A. B. (2008). Ultrasound emulsification: effect of ultrasonic and physicochemical properties on dispersed phase volume and droplet size. Ultrasonics Sonochemistry, 15(4), 554 563.

Galazka, V. B., Dickinson, E., \& Ledward, D. A. (1996). Effect of high pressure on the emulsifying behaviour of $\beta$-lactoglobulin. Food Hydrocolloids, 10, 213-219.

Gharibzahedi, S. M. T., Mousavi, S. M., Hamedi, M., Khodaiyan, F., \& Razavi, S. H. (2012). Development of an optimal formulation for oxidative stability of walnut-beverage emulsions based on gum arabic and xanthan gum using response surface methodology. Carbohydrate Polymers, 87(2), 1611-1619.

Goodwin, D. J., Picout, D. R., Ross-Murphy, S. B., Holland, S. J., Martini, L. G., \& Lawrence, M. J. (2011). Ultrasonic degradation for molecular weight reduction of pharmaceutical cellulose ethers. Carbohydrate Polymers, 83(2), 843-851.

Huang, X., Kakuda, Y., \& Cui, W. (2001). Hydrocolloids in emulsions: particle size distribution and interfacial activity. Food Hydrocolloids, 15(4-6), 533-542.

Huck-Iriart, C., Álvarez-Cerimedo, S., Candal, R. J., \& Herrera, M. L. (2011). Structures and stability of lipid emulsions formulated with sodium caseinate. Current Opinion in Colloid Interface Science, $16(5), 412-420$.

Jafari, S. M., Assadpoor, E., He, Y., \& Bhandari, B. (2008). Recoalescence of emulsion droplets during high-energy emulsification. Food Hydrocolloids, 22, 1191-1202.

Jafari, S. M., He, Y., \& Bhandari, B. (2006). Nano-emulsion production by sonication and microfluidization - a comparison. International Journal of Food Properties, 9(3), 475-485.

Jiménez, A., Beltrán, G., \& Uceda, M. (2007). High-power ultrasound in olive paste pretreatment. Effect on process yield and virgin olive oil characteristics. Ultrasonics Sonochemistry, 14(6), 725-731.

Kasaai, M. R., Arul, J., \& Charlet, G. (2008). Fragmentation of chitosan by ultrasonic irradiation. Ultrasonics Sonochemistry, 15(6), 10011008.

Kang, K. S., \& Pettitt, D. J. (1993). Xanthan, gellan, welan, rhamsam. In R. L. Whistler \& J. N. BeMiller (Eds.), Industrial gums: polysaccharides and their derivatives (3rd ed., pp. 341-371). New York, USA: Academic Press.

Karaman, S., Tahsin Yilmaz, M., Ertugay, M. F., Baslar, M., \& Kayacier, A. (2012). Effect of ultrasound treatment on steady and dynamic shear properties of glucomannan based salep dispersions: optimization of amplitude level, sonication time and temperature using response surface methodology. Ultrasonics Sonochemistry, 19(4), 928-938.

Karbstein, H., \& Schubert, H. (1995). Developments in the continuous mechanical production of oil-in-water macro-emulsions. Chemical Engineering and Processing Process Intensification, 34(3), 205211.

Kentish, S., Wooster, T. J., Ashokkumar, M., Balachandran, S., Mawson, R., \& Simons, L. (2008). The use of ultrasonics for nanoemulsion preparation. Innovative Food Science and Emerging Technologies, 9, 170-175.

Krstonošić, V., Dokić, L., Dokić, P., \& Dapčević, T. (2009). Effects of xanthan gum on physicochemical properties and stability of corn oilin-water emulsions stabilized by polyoxyethylene (20) sorbitan monooleate. Food Hydrocolloids, 23(8), 2212-2218.

Leroux, J., Langendorff, V., Schick, G., Vaishnav, V., \& Mazoyer, J. (2003). Emulsion stabilizing properties of pectin. Food Hydrocolloids, 17, 455-462.

Li, M. K., \& Fogler, H. S. (1978). Acoustic emulsification. Part 1. The instability of the oil-water interface to form the initial droplet. Journal of Fluid Mechanics, 88, 499-512.
Li, P. H., \& Chiang, B. H. (2012). Process optimization and stability of Dlimonene-in-water nanoemulsions prepared by ultrasonic emulsification using response surface methodology. Ultrasonics Sonochemistry, 19(1), 192-197.

Lorimer, J. P., Mason, T. J., Cuthbert, T. C., \& Brookfield, E. A. (1995). Effect of ultrasound on the degradation of aqueous native dextran. Ultrasonics Sonochemistry, 2, 55-57.

Marcotte, M., Taherian, A. R., \& Ramaswamy, H. S. (2001). Evaluation of rheological properties of selected salt enriched food hydrocolloids. Journal of Food Engineering, 48(2), 157-167.

McClements, D. J. (2005). Food emulsions: principles, practice, and techniques. Boca Raton, FL: CRC Press.

Mengual, O., Meunier, G., Cayre, I., Puech, K., \& Snabre, P. (1999). Characterisation of instability of concentrated dispersions by a new optical analyser: the TURBISCAN MA 1000. Colloids and Surfaces A: Physicochemical and Engineering Aspects, 152, 111123.

Milas, M., Rinaudo, M., \& Tinlands, B. (1986). Comparative depolymerization of xanthan gum by ultrasonic and enzymic treatments. Rheological and structural properties. Carbohydrate Polymers, 6, 95-107.

Moncada Reyes ML (2011) Influence of low sonication intensities at different temperatures on acid tolerance, bile tolerance, protease ativity, and growth of yogurt culture bacteria Lactobacillus delbrueckii spp. Bulgaricus and Streptococcus salivarius ssp. Thermophilus. Master Thesis. School of Animal Science, Louisiana State University, Louisiana, USA.

Muthukumaran, S., Kentish, S. E., Stevens, G. W., \& Ashokkumar, M. (2006). Application of ultrasound in membrane separation processes: a Review. Reviews in Chemical Engineering, 22, 155-194.

Olson, D. W., White, C. H., \& Richter, R. L. (2004). Effect of pressure and fat content on particle sizes in microfluidized milk. Journal of Dairy Science, 87(10), 3217-3223.

Palazolo, G. G., Sorgentini, D. A., \& Wagner, J. R. (2004). Emulsifying properties and surface behavior of native and denatured whey soy proteins in comparison with other proteins. Creaming stability of oil in-water emulsions. Journal of American Oil Chemists' Society, $81(7), 625-632$.

Palazolo, G. G., Sorgentini, D. A., \& Wagner, J. R. (2005). Coalescence and flocculation in $\mathrm{O} / \mathrm{W}$ emulsions of native and denatured whey soy proteins in comparison with soy protein isolates. Food Hydrocolloids, 19, 595-604.

Pizzino, A., Catte, M., Van Hecke, E., Salager, J. L., \& Aubry, J. M. (2009). On-line light backscattering tracking of the transitional phase inversion of emulsions. Colloids and Surfaces A: Physicochemical and Engineering Aspects, 338, 148-154.

Price, G. (1990). The use of ultrasound for the controlled degradation of polymer solutions. In T. J. Mason (Ed.), Advances in sonochemistry (pp. 231-287). Greenwich, CT: JAI Press.

Rawle, A. (2002). The importance of particle sizing to the coatings industry. Advances in Colour Science and Technology, 5(1), 1-12.

Relkin, P., \& Sourdet, S. (2005). Factors affecting fat droplet aggregation in whipped frozen protein-stabilized emulsions. Food Hydrocolloids, 19, 503-51.

Santos, H. M., Lodeiro, C., \& Capelo-Martinez, J.-L. (2009). The power of ultrasound. In J.-L. Capelo-Martinez (Ed.), Chemistry: analytical applications (pp. 1-16). KGaA, Weinheim, Germany: Wiley-VCH Verlag $\mathrm{GmbH} \& \mathrm{Co}$.

Stellbrink, J., Abbas, B., Allgaier, J., Monkenbusch, M., Richter, D., Likos, C. N., Löwen, H., \& Watzlawek, M. (1998). Structure and dynamics of star polymers. Progress Colloid Polymer Science, 28, $110-125$.

Striegel, A. M. (2003). Influence of chain architecture on the mechanochemical degradation of macromolecules. Journal of Biochemical and Biophysical Methods, 56, 117-139. 
Surh, J., Decker, E. A., \& McClements, D. J. (2006). Influence of $\mathrm{pH}$ and pectin type on properties and stability of sodiumcaseinate stabilized oil-in-water emulsions. Food Hydrocolloids, 20(50), 607-618.

Syed Gulrez, K. H., Al-Assaf, S., Fang, Y., Phillips, G. O., \& Gunning, A. P. (2012). Revisiting the conformation of xanthan and the effect of industrially relevant treatments. Carbohydrate Polymers, 90(3), 1235-1243.

Tang, S. Y., Manickam, S., Wei, T. K., \& Nashiru, B. (2012). Formulation development and optimization of a novel Cremophore EL-based nanoemulsion using ultrasound cavitation. Ultrasonics Sonochemistry, 19, 330-345.

Tang, S. Y., Shridharan, P., \& Sivakumar, M. (2013). Impact of process parameters in the generation of novel aspirin nanoemulsionsComparative studies between ultrasound cavitation and microfluidizer. Ultrasonics Sonochemistry, 20, 485-497.

Tayal, A., \& Khan, S. A. (2000). Degradation of a water-soluble polymer: molecular weight changes and chain scission characteristics. Macromolecules, 33, 9488-9493.

Tiwari, B. K., Muthukumarappan, K., O’Donnell, C. P., \& Cullen, P. J. (2010). Rheological properties of sonicated guar, xanthan and pectin dispersions. International Journal of Food Properties, 13, 223-233.

Tonon, R. V., Grosso, C. R. F., Míriam, D., \& Hubinger, M. (2011). Influence of emulsion composition and inlet air temperature on the microencapsulation of flaxseed oil by spray drying. Food Research International, 44, 282-289.

Vinod, V. T. P., \& Sashidhar, R. B. (2009). Solution and conformational properties of gum kondagogu (Cochlospermum gossypium) - a natural product with immense potential as a food additive. Food Chemistry, 116(3), 686-692.

Walstra, P. (2003). Physical chemistry of foods. New York, USA: Marcel Dekker.

Wasikiewicz, J. M., Yoshii, F., Nagasawa, N., Wach, R. A., \& Mitomo, H. (2005). Degradation of chitosan and sodium alginate by gamma radiation, sonochemical and ultraviolet methods. Radiation Physics and Chemistry, 73, 287-295.

Wu, H., Hulbert, G. J., \& Mount, J. R. (2000). Effects of ultrasound on milk homogenization and fermentation with yogurt starter. Innovative Food Science and Emerging Technologies, 1, 211-218.

Zhou, Y., \& Shi, J. (2006). Effects of extracellular calcium on cell membrane resealing in sonoporation. Journal of Controlled Release, 126, 34-43.

Zisu, B., Bhaskaracharya, R., Ashokkumar, M., \& Kentish, S. (2010). Ultrasonic processing of dairy systems in large scale reactors. Ultrasonics Sonochemistry, 17(6), 1075-1081.

Zúñiga, R. N., Skurtys, O., Osorio, F., José, M., Aguilera, J. M., \& Pedreschi, F. (2013). Optical properties of emulsion-based hydroxypropyl methylcellulose (HPMC) films: effect of their microstructure. InsideFood Symposium, 90(2), 1147-1158. 